\title{
High- and low-dose OPG-Fc cause osteopetrosis-like changes in infant mice
}

\author{
Renee Bargman 1,2, Ram Posham ${ }^{3}$, Adele Boskey ${ }^{3,4}$, Erin Carter ${ }^{5}$, Edward DiCarlo ${ }^{6}$, Kostas Verdelis ${ }^{3}$, \\ Cathleen Raggio ${ }^{3,5}$ and Nancy Pleshko $0^{3,7}$
}

BACKGROUND: Receptor activator of nuclear factor-kB ligand (RANKL) inhibitors are being considered for use in children with osteogenesis imperfecta $(\mathrm{OI})$. We sought to assess efficacy of two doses of a RANKL inhibitor, osteoprotegerinimmunoglobulin Fc segment complex (OPG-Fc), in a growing animal model of Ol, the col1 a2-deficient mouse (oim/oim) and its wild-type controls (+/+).

METHODS: Treated mice showed runting and radiographic evidence of osteopetrosis with either high- $(20 \mathrm{mg} / \mathrm{kg}$ twice weekly) or low-dose (1 mg/kg/week) OPG-Fc. Because of this adverse event, OPG-Fc treatment was halted, and the mice were killed or monitored for recovery with monthly radiographs and assessment of serum osteoclast activity (tartrateresistant acid phosphatase $5 b$, TRACP-5b) until 25 wk of age.

RESULTS: Twelve weeks of OPG-Fc treatment resulted in radiographic and histologic osteopetrosis with no evidence of bone modeling and negative tartrate-resistant acid phosphatase staining, root dentin abnormalities, and TRACP-5b activity suppression. Signs of recovery appeared 4-8 wk post-treatment.

CONCLUSION: Both high- and low-dose OPG-FC treatment resulted in osteopetrotic changes in infant mice, an outcome that was not seen in studies with the RANKL inhibitor RANKimmunoglobulin Fc segment complex (RANK-Fc) or in studies with older animals. Further investigations of RANKL inhibitors are necessary before their consideration for use in children.

$B^{\circ}$ one remodeling involves bone resorption by osteoclasts and bone synthesis by osteoblasts. Therapies based on osteoclast suppression are increasingly used in children with disorders of bone metabolism (1). Bisphosphonates are increasingly used in children with moderate to severe osteogenesis imperfecta (OI) (1). However, recent studies of bisphosphonate therapy in adults and, in some cases, children, report concerns about oversuppression of bone turnover, hypocalcemia, osteonecrosis, and depression of inflammatory responses $(1,2)$. Additional complications unique to the pediatric population are emerging, such as delayed healing of osteotomies (3) and fractures (4). One study reported osteopetrosis-like changes in a child without underlying bone disease treated with long-term, high-dosage bisphosphonates (5). Therefore, alternative therapeutic options are being explored.

Interaction between receptor activator of nuclear factor- $\kappa \mathrm{B}$ (RANK) and its ligand (RANKL) is essential for osteoclast formation, function, and survival (6). Osteoprotegerin (OPG) is a factor that binds RANKL, inhibiting RANK/RANKL interaction (6). Recent therapies for osteoporosis and related conditions of decreased bone density have targeted the RANK/RANKL pathway. Multiple studies in humans and animal models have shown that interference with RANKL signaling suppresses bone resorption and increases bone volume, density, and strength (6).

Denosumab is a human monoclonal immunoglobulin G2 anti-RANKL antibody approved by the US Food and Drug Administration for clinical use in adults who have metastatic disease to bone or osteoporosis $(7,8)$. In contrast to bisphosphonates, which remain in the bone for decades (2), denosumab does not incorporate into the bone matrix (6). This is a desirable attribute of therapy for long-term use and for use in children, but to date, it has not been evaluated clinically in a pediatric population. Therefore, the long-term effects of this therapy in children are not known. In addition to concerns over growing bone development, there are concerns that, because denosumab has immune system effects, immune or malignant issues may arise as they did in RANKL knockout mice with lymph node agenesis (9).

The efficacy of denosumab is limited in most animal studies as it recognizes only primate RANKL(10), and OPG and/ or soluble RANK-immunoglobulin $\mathrm{Fc}$ segment complex (RANK-Fc) have been used as surrogates for denosumab in many animal studies of RANKL inhibition (11-19). Transgenic mice overexpressing OPG or RANK-Fc have been shown to develop osteopetrosis (20), a skeletal disorder of osteoclast function secondary to either an absence of osteoclasts or their intrinsic defects that leads to skeletal deformity, increased skeletal mass, and increased fracturing (21). In the growing individual, osteopetrosis is characterized by the persistence of cartilaginous tissue in regions of endochondral ossification and the failure to model bone. Mouse models of osteopetrosis, such as the $o p / o p$ mice, completely lack tooth eruption (22)

\footnotetext{
'Department of Pediatrics, New York Presbyterian Hospital, Weill Cornell Medical Center, New York, New York; ${ }^{2}$ Department of Pediatrics, Nassau University Medical Center, East Meadow, New York; ${ }^{3}$ Research Division, Hospital for Special Surgery, New York, New York; ${ }^{4}$ Department of Physiology, Biophysics and Systems Biology, and

Department of Biochemistry, Weill Medical College of Cornell University, New York, New York; ${ }^{5}$ Center for Skeletal Dysplasias, Hospital for Special Surgery, New York, New York;

${ }^{6}$ Department of Pathology, Hospital for Special Surgery, New York, New York; ${ }^{7}$ Department of Bioengineering, Temple University, Philadelphia, Pennsylvania.

Correspondence: Nancy Pleshko (npleshko@temple.edu)
} 
because functional osteoclasts are essential for bone remodeling that allows tooth eruption (23).

Animal studies of RANKL inhibition, including those in mice, rats, pigs, and monkeys, have used a range of doses of human OPG, OPG-Fc, and RANK-Fc. To date, none have reported the development of osteopetrotic features $(11-19,24)$, although the majority of the studies have not initiated treatment in neonatal animals. As it is a disease that develops during growth, evaluation of treatment with antiresorptive agents in neonatal animals is of interest.

In this study, we report the appearance of osteopetrotic features in infantile wild-type (+/+) and oim/oim mice, a model of moderate-to-severe OI (25), treated with low and high doses of OPG-Fc. These results have immediate implications for clinical treatment of very young children with RANKL inhibitors.

\section{RESULTS}

\section{Runting}

Although the animals gained weight during the course of treatment, OPG-Fc-treated animals generally gained less as compared with saline-treated controls (gender and genotypematched). Results are presented for the groups of $+/+$ and oim/ oim saline controls and for individual animals in the treated case reports (Table 1). One oim/oim low-dose OPG-treated mouse died during the recovery phase of unknown causes.

\section{Serum Chemistry}

Tartrate-Resistant Acid Phosphatase 5b. After 4 wk of OPG-Fc treatment, average tartrate-resistant acid phosphatase 5b (TRACP-5b) activity was reduced by $75 \%$ in the high-dose group and $64 \%$ in the low-dose group as compared with salinetreated controls $(0.50 \pm 0.35,0.71 \pm 0.56$, and $1.99 \pm 0.42 \mathrm{U} / \mathrm{l}$, respectively) (Table 1 ). At 25 wk of age, some mice had increased serum TRACP-5b levels, indicative of recovering osteoclast activity, whereas others did not.

\section{Radiographic Analyses}

After $4 \mathrm{wk}$ of high- or low-dose OPG-Fc treatment, mice had shortened club-shaped long bones, undermodeled diaphyses, with increased metaphyseal radiographic intensity as compared with the saline-treated controls (Figure 1a,e,i,m and Figure 2). One $+/+$ male treated for $9 \mathrm{wk}$ with high-dose OPG-Fc had two fractures. All oim/oim mice had fractures regardless of treatment.

\section{Histology}

For saline-treated +/+ animals, histologic evaluation demonstrated normal remodeling activity and structure as previously reported (26) (Figure $\mathbf{1 b}, \mathbf{c}$ ). Oim/oim mice had thin cortices, and metaphyseal trabeculae were thin and fewer in number (Figure 1 $\mathbf{j}, \mathbf{k}$ ). The presence and distribution of osteoclasts, as demonstrated by tartrate-resistant acid phosphatase staining, were appropriate for each genotype (Figure 1d,1).

The OPG-Fc-treated mice that received the high dose for $12 \mathrm{wk}$ showed nearly complete retention of growth-plate cartilage and bone in the metaphyses, resulting in a fusiform deformity of the bone without modeling of the distal femur, both features typical of osteopetrotic bone (Figure 1f,g,n,o). The $+/+$ mice had more bone as compared with oim/oim mice, whereas the oim/ oim mice had less bone and more residual calcified cartilage (primary spongiosa). In all OPG-Fc-treated mice, the marrow space was reduced because of the persistence of the unresorbed physeal cartilage in the primary spongiosa, features that are indistinguishable from osteopetrosis (21). Tartrate-resistant acid phosphatase staining showed a complete absence of osteoclasts in these mice (Figure 1h,p). Figure 1 shows typical features in two mice, but the entire group exhibited similar findings.

\section{Dental Evaluation}

The rate of murine incisor eruption is equal to the rate of wear, such that the incisors remain at a constant size in adult mice. Incisors from saline-treated control mice demonstrated signs of normal incisor development and eruption. The enamel-free segment near the root apex reflects the normal process of odontogenesis wherein enamel develops following formation of a substantial layer of dentin (Figure 3a). Incisors from all genotypes treated with OPG-Fc showed arrest of incisor eruption. In the oim/oim mice, arrest of incisor eruption was accompanied by pronounced thickening of dentin and constriction of the pulp space throughout the root (Figure 3b). The enamel layer was also thickened and extended to the apical end of the root follicle. The root follicle was narrow and did not extend as far distally into the jaw as compared with those of saline-treated controls. The $+/+$ mice treated with OPG-Fc showed thickened dentin and enamel layers, but a more open and extensive follicle (Figure 3c). All OPG-Fc-treated mice also presented several sites of ankylosis between the alveolar bone and the root where the periodontal ligament was severely restricted or absent (Figure 3d).

\section{Recovery}

Recovery of bone remodeling, based on visual inspection of the faxitron radiographic intensity, was variable in extent, even among mice of the same sex and genotype that received identical treatment (Figure 4). In general, mice treated with lower doses of OPG-Fc for shorter lengths of time appeared to recover faster.

\section{DISCUSSION}

This study reports osteopetrotic features in the mineralized tissue of infantile $+/+$ and oim/oim mice after RANKL inhibition with OPG-Fc. The finding is not an entirely unexpected complication of RANKL inhibition in the very young (20). Transgenic mice expressing a soluble RANK-Fc fusion protein have severe osteopetrosis because of a reduction in osteoclasts (20), and transgenic mice overexpressing OPG show similar findings (27). Similar abnormalities have also been reported in both animal and human subjects given high-dose bisphosphonates $(5,28,29)$. Therefore, the finding serves as a caution in the treatment of young children.

It is unlikely that the osteopetrosis-like changes seen here were solely because of the dosage of OPG-Fc, as similar doses have been used previously in older animals with no ill effects $(11,13,15-17,19)$. We hypothesize that treatment in the neonatal 
Table 1. Weight and osteoclast function (serum TRACP-5b U/I) in OPG-Fc and saline-treated mice

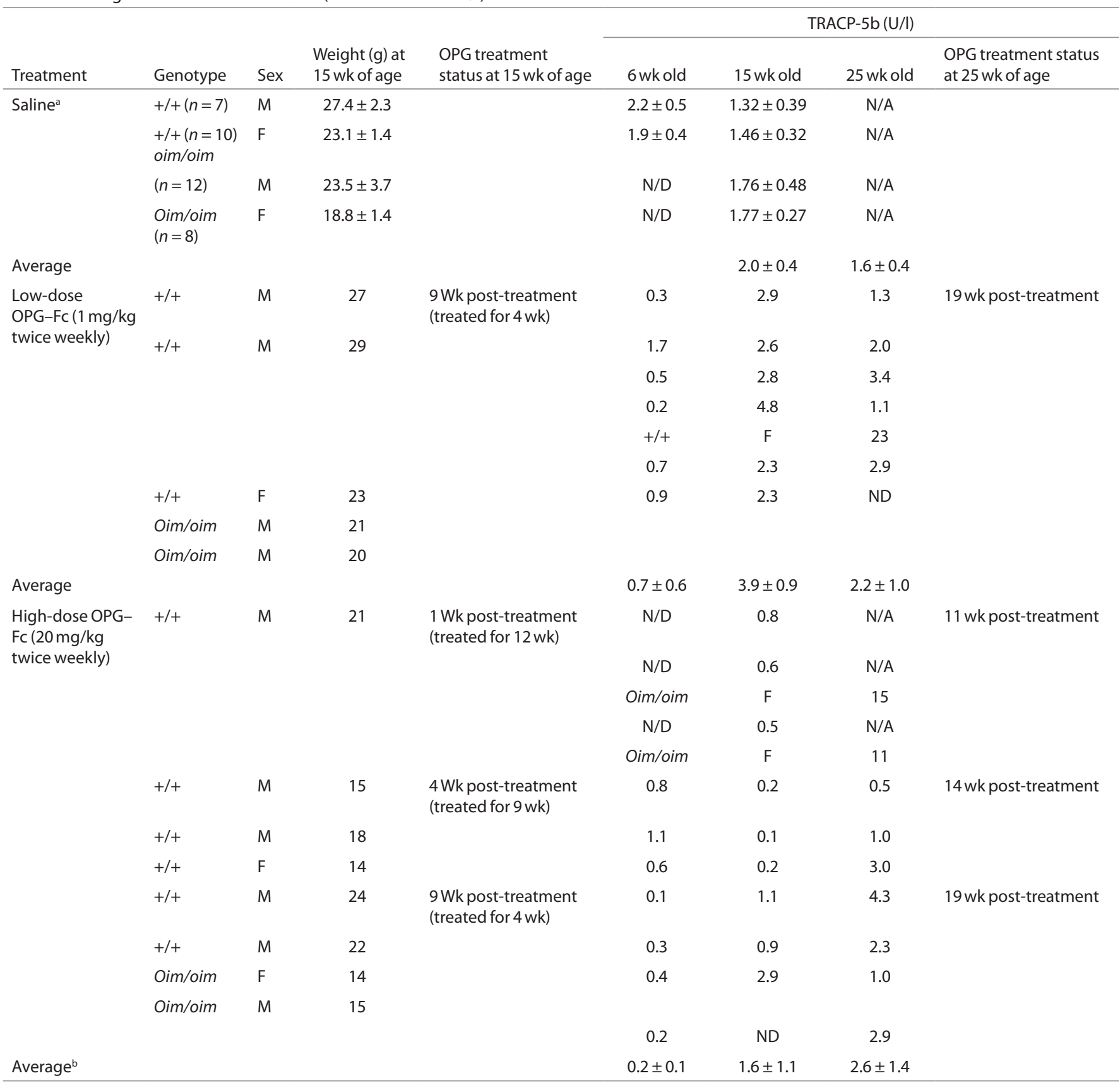

Treatments were initiated at 2 wk of age. All saline-treated mice and the initial group of high-dose mice were killed at 15 wk of age. For saline oim/oim, the entire group was not assayed, and the initial group of high-dose mice was not assayed at 6 wk; for OPG treatment, one mouse died before the specimen could be collected.

N/A, not available; ND, not determined; OPG-Fc, osteoprotegerin-immunoglobulin Fc segment complex; TRACP-5b, tartrate-resistant acid phosphatase 5b.

aSaline-treated $+/+$ and oim/oim are presented as averages \pm SD. ${ }^{b}$ Average of $9 \mathrm{wk}$ post-treatment (treated for $4 \mathrm{wk}$ ).

period is a key factor in the development of these osteopetrotic changes. The low dose of $1 \mathrm{mg} / \mathrm{kg} / \mathrm{wk}$ is actually lower than any of the published doses used in animals, and is consistent with the dose used in clinical trials in adult patients with osteoporosis (7). Before this report, the youngest animals to be treated with OPG-Fc were 5-7 wk old and received a dose of $10 \mathrm{mg} /$ $\mathrm{kg} / \mathrm{dose}$ three times weekly with no reported adverse effects (30). Young animals depend on their osteoclasts for modeling bone, and therefore they are more sensitive to osteoclast inhibition. The specific RANKL inhibitor used is also a factor in the extent of remodeling inhibition. The recent study from our laboratory that used a different RANKL inhibitor to treat neonatal oim/oim mice, RANK-Fc (24), found reduced fracture incidence and no mineralization abnormalities.

Bisphosphonate-induced osteopetrosis has been described in both animal models and clinically in humans $(5,28,29,31,32)$. This seems to occur only when treatment is initiated in young subjects. Animal studies using bisphosphonates showed similar 


\section{Articles | Bargman et al.}

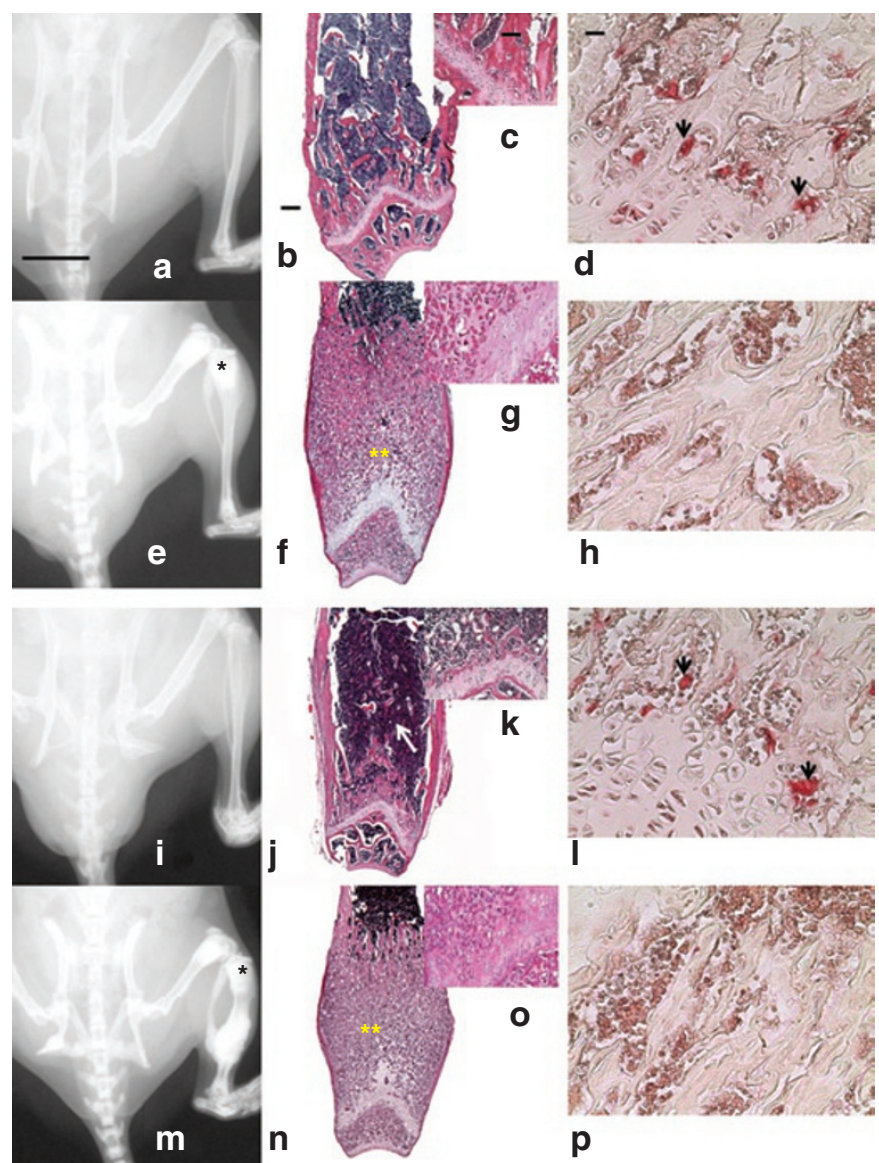

Figure 1. Radiographic and histological findings of 15 -wk-old mice after 12 wk of treatment with either saline or high-dose osteoprotegerin-immunoglobulin Fc segment complex (OPG-Fc). Radiologically and histologically apparent osteopetrosis-like features including increased metaphyseal density $\left({ }^{*}\right)$, persistence of calcified cartilage (primary spongiosa) without evidence of bone remodeling $\left(^{* *}\right)$, and compromise of the marrow space in both $+/+$ and oim/oim mice treated with OPG-Fc. Tartrate-resistant acid phosphatase (TRAP) staining showed the presence of osteoclasts (black arrows) in saline-treated animals but absence of osteoclasts in OPG-Fc-treated animals. +/+ saline: (a) radiograph (scale bar $=10 \mathrm{~mm}$ ); (b) hematoxylin and eosin (H\&E) $2.5 \times(\mathrm{scale}$ bar $=250 \mu \mathrm{m})$; (c) H\&E 20X (scale bar $=100 \mu \mathrm{m})$; (d) TRAP staining 40X (scale bar $=50 \mu \mathrm{m}$ ). Black arrows denote osteoclasts. +/+ high-dose OPG-Fc: (e) radiograph; (f) H\&E 2.5X; (g) H\&E 20x; (h) TRAP staining 40x. oim/oim saline: (i) radiograph; (j) H\&E 2.5 $\times$, thinner trabeculae in comparison with +/+ animals are noted (white arrow); (k) H\&E 20x; (I) TRAP staining 40x. Black arrows denote osteoclasts. oim/oim high-dose OPG-Fc: (m) radiograph; (n) H\&E 2.5X; (o) H\&E 20x; (p) TRAP staining 40X.

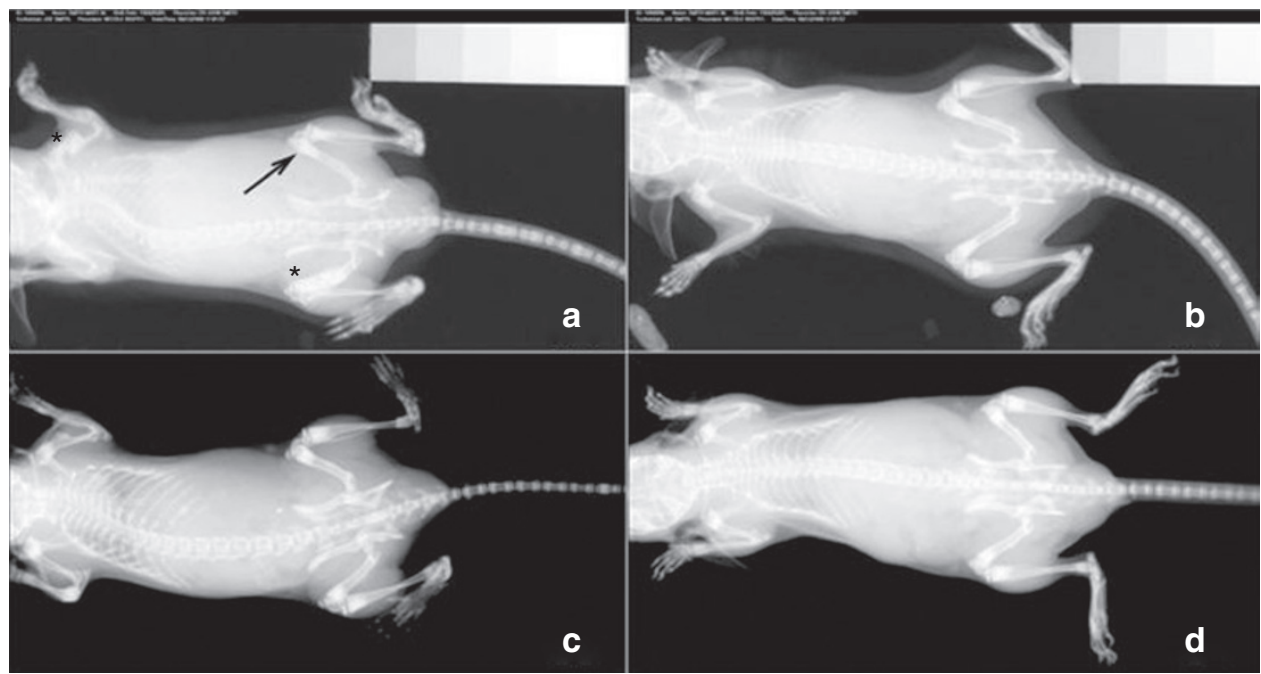

Figure 2. Faxitron radiographs of 6-wk-old osteoprotegerin-immunoglobulin Fc segment complex (OPG-Fc)-treated mice. (a) Oim/oim treated with low-dose OPG-Fc for 4 wk. (b) +/+ treated with low-dose OPG-Fc for 4 wk. (c) Oim/oim treated with high-dose OPG-Fc for 4 wk. (d) $+/+$ treated with high-dose OPG-Fc for 4 wk. High-intensity unremodeled metaphyseal bone (shown with an arrow in (a)) is present in all treated animals. Fractures were evident in the treated oim/oim mice (shown with asterisks in (a)). The density calibration standard is shown in (a) and (b). 

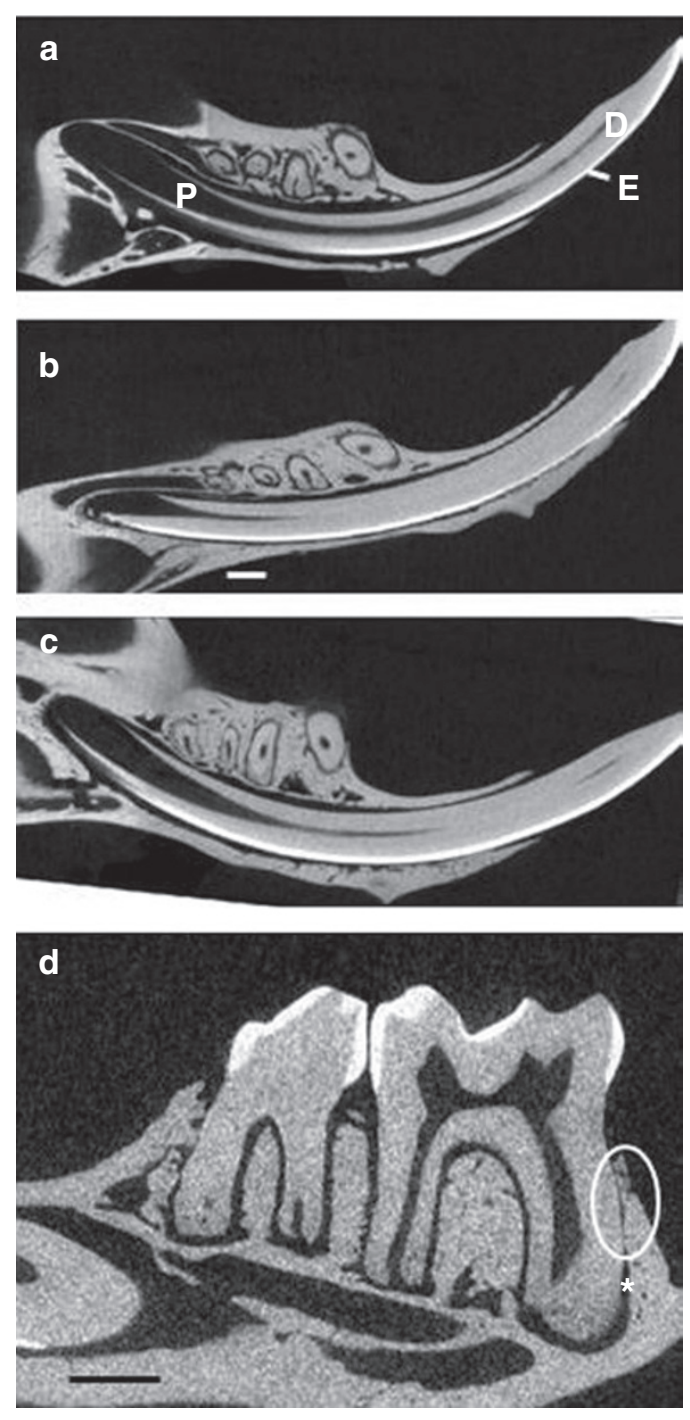

Figure 3. Microcomputed tomography (micro-CT) images of hemimandibles of 15 -wk-old mice treated for 12 wk as follows: (a) +/+ treated with saline; (b) oim/+ treated with high-dose OPG-Fc showing an arrest of eruption of the incisor (scale bar $=0.5 \mathrm{~mm}$ ); and $(\mathbf{c})+/+$ treated with highdose OPG-Fc showing an intermediate incisor phenotype. Enamel, dentin, and pulp are noted in (a) $(E=$ enamel, $D=$ dentin, and $P=$ pulp). (d) Higher resolution (voxel size $=6 \mu \mathrm{m}$ ) micro-CT image of molars from oim $/+$ mouse treated with high-dose OPG-Fc (scale bar $=0.5 \mathrm{~mm}$ ). Area of ankylosis between the alveolar bone and the root is indicated by the circle. Severe restriction of the periodontal ligament is denoted by the asterisk.

changes to those seen in this study: decreased bone growth and weight gain, persistence of calcified cartilage, bone deformities, and increased susceptibility to fracture $(29,32)$. One study reported similar findingsin a child with bisphosphonate-induced osteopetrosis, including dense metaphyses and club-shaped deformities of the long bones on radiography and unresorbed calcified cartilage on histology as well as metaphyseal osteopenia and frequent fractures after cessation of therapy (33). An important distinction is that bisphosphonate-induced osteopetrosis has been reported in cases in which the doses used were higher than what is considered therapeutic $(5,28,29,31-$ 32), whereas we observed osteopetrotic features with OPG-Fc at doses that were otherwise therapeutic in adult animals and human clinical trials. Therefore, OPG-Fc appears to be a more potent inhibitor of osteoclasts than bisphosphonates, and although OPG-Fc will not be used in humans, care needs to be taken with denosumab as well. Although this may be beneficial in treating adults with osteoporosis, in children, who grow more rapidly during infancy as compared with other periods of life, it may be difficult to regulate the dosage required for a sufficient but not excessive degree of osteoclast inhibition.

The dental abnormalities observed in this study are also interesting, and consistent with those seen in osteopetrosis. In the mice treated with OPG-Fc for $12 \mathrm{wk}$, dental abnormalities included arrest of incisor growth and focal ankylosis of the root. The defects in tooth development observed in the OPG-Fctreated mice were in agreement with previous observations on bisphosphonate-induced $(29,34)$ or inherited osteopetrosis $(35,36)$ in mice. This slowing of incisor growth, which also resulted in a shortened incisor tip, is most likely due to a slowing of bone resorption in the dental crypt and subsequent failure of further root eruption as previously described. However, we did not observe a thinning of the enamel or disorganization of the dentin layer in the small number of studied animals, as has been reported in bisphosphonate-induced osteopetrosis in mice (29).

It is unlikely that the runting in OPG-Fc treated mice was because of the dental phenotype, i.e., an inability to eat. The mice in this study began OPG-Fc treatment after the completion of tooth eruption, reported by day 14 of life (37), so these mice would not be expected to have problems with tooth eruption. However, we did not evaluate the possibility that a delay in incisor growth may result in some degree of malocclusion. Some children treated with bisphosphonates appear to have delayed tooth eruption (38), indicating that osteoclast inhibition causes dental changes even in the absence of frank osteopetrosis.

The primary limitation of this report is the small number of mice reported. Due to the severity of the adverse reaction in the animals, continuation of the OPG-Fc arm of the study could not be justified, and we were not able to obtain large enough numbers for statistical analysis. Therefore, the complex timing and dosing issues that surround the use of the RANKL inhibitor OPG-Fc in the infant oim/oim mouse could not be addressed. Nevertheless, osteopetrotic features were observed in every mouse that was given either high- or low-dose OPG-Fc. Although recovery toward normal remodeling and osteoclast activity was evident as early as $4 \mathrm{wk}$ after cessation of the treatment, together, these data clearly support the importance of further investigation of these agents before use in young children.

\section{METHODS}

\section{Animals}

All procedures were approved by the IACUC at the Hospital for Special Surgery. Breeding animals of mixed homozygous oim/oim, heterozygous oim/t, and wild-type $(+/+)$ genotypes (with C57BL/6J backgrounds) were obtained from Jackson Laboratories (Bar Harbor, ME). Their offspring were used in this study to assess the potential of the RANKL inhibitor OPG-Fc (supplied by Amgen, Thousand Oaks, CA) to improve bone properties in oim/oim mice. Although OPG-Fc is not used clinically, its use to treat mice in this study is analogous to the use of denosumab in humans. Oim/oim mice display a moderate-to-severe 


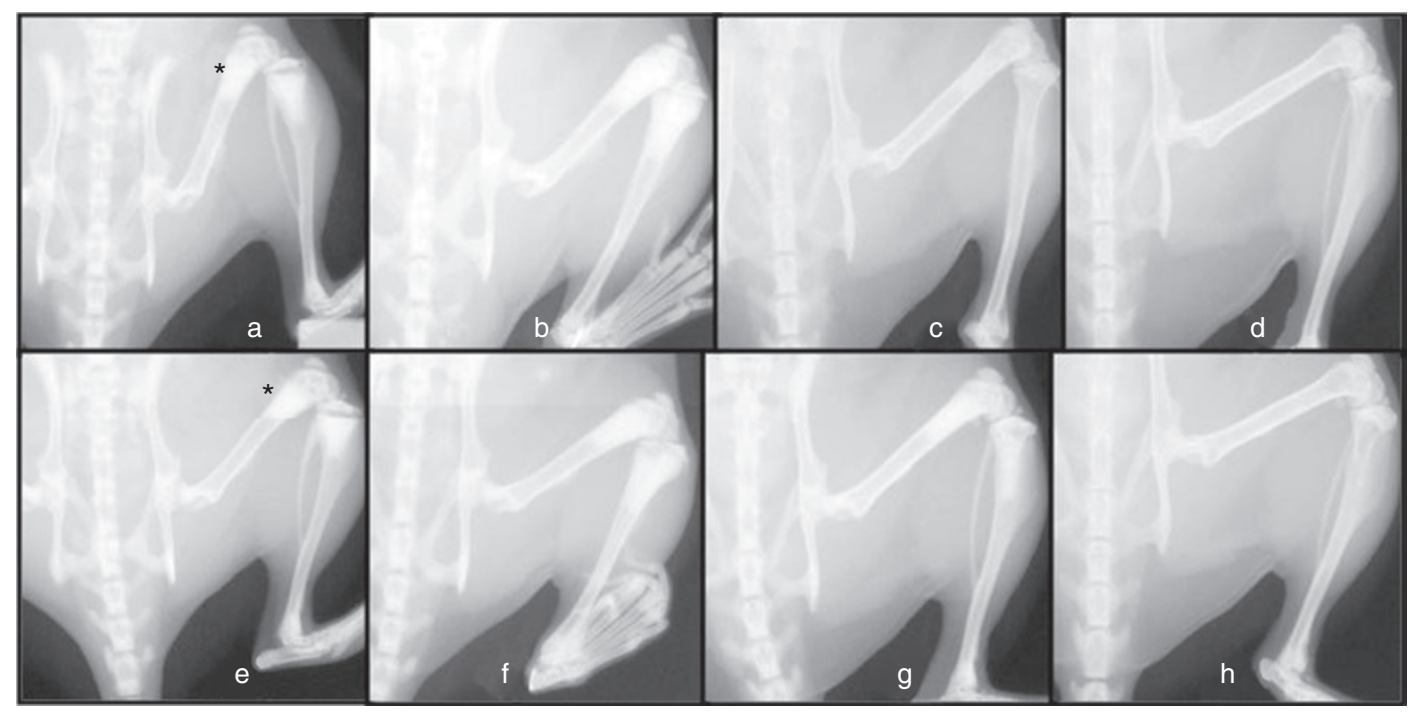

Figure 4. Radiographs of two +/+ mice treated with high-dose OPG-Fc for $4 \mathrm{wk}$, demonstrating tartrate-resistant acid phosphatase recovery (increased serum tartrate-resistant acid phosphatase $5 \mathrm{~b}$ (TRACP-5b) values) at $10 \mathrm{wk}$ of age and radiologic recovery by 14 wk in the first case (top row, (a-d)), and by 14 and 18 wk, respectively, in the second case (bottom row, (e-h)). Asterisks (a,e) denote initial regions of increased metaphyseal density at 6 wk of age, which eventually return to normal density. Age of mouse and serum TRACP-5b values (U/l): (a) 6 wk, 0.49; (b) 10 wk, 2.75; (c) 14 wk, 2.84; (d) 18 wk, 1.42; (e) $6 \mathrm{wk}, 0.21$; (f) $10 \mathrm{wk}, 0.42 ;$ (g) $14 \mathrm{wk}, 4.83$; and (h) $18 \mathrm{wk}, 2.47$.

OI phenotype (although they differ from most human patients with OI in that they synthesize only homotrimeric type I collagen as has been found in at least one case of human OI) (39) and typically sustain one to seven fractures during their growth period (26), whereas oim/+ mice have a mild OI phenotype and only occasionally sustain a fracture. The control arm of the study received saline $(n=17+/+$ and 20 oim/oim). The initial study design based on a power analysis required 20 mice per group with the goal of assessment of two different doses of OPG-Fc at the end of a 12 -wk treatment. Animals were weaned at $3 \mathrm{wk}$ of age, after which they were grouped by sex, housed up to six animals per cage in a light-controlled environment $(12 \mathrm{~h}$ light-dark cycles), maintained on the same treatment, and given autoclaved water and whole and powdered Rodent Diet (Purina, St Louis, MO).

Infant oim/oim, oim/t, and wild-type $(+/+)$ mice $(\sim 2 \mathrm{wk}$ old $)$ were treated with OPG-Fc at a high dose (20 mg/kg/twice/wk) (40), a low dose $(1 \mathrm{mg} / \mathrm{kg} / \mathrm{wk})$, or with saline. Genotyping was done at the end of the study. Treatment was initiated in groups of littermates in a staggered manner as they became available at the age of $2 \mathrm{wk}$. This meant that the treatments of some litters began weeks after previous litters had begun treatment. Mice were weighed twice weekly to determine appropriate drug dose. When the first group of mice treated with high-dose OPG-Fc reached the age of $15 \mathrm{wk}$ ( $12 \mathrm{wk}$ of treatment, $n=2 \mathrm{oim} /+, n=2 \mathrm{oim} / \mathrm{oim}$, and $n=1+/+)$, significant runting based on weight gain, observation, and radiographs was observed in all genotypes. These five mice were killed for histological analysis. This adverse event halted the administration of both doses of OPG-Fc in all animals regardless of how long they had been treated. As the number of weeks the mice had been treated varied when the decision to stop OPG-Fc was made, this resulted in case studies of different lengths of treatment of OPG-Fc (high-dose treatment for $9 \mathrm{wk}$ : $n=3+/+$ and $n=3 \mathrm{oim} /+$; high-dose treatment for $4 \mathrm{wk}: n=2+/+, n=$ $1 \mathrm{oim} /+$, and $n=2 \mathrm{oim} / \mathrm{oim}$; low-dose treatment for $4 \mathrm{wk}: n=4+/+, n=$ $4 \mathrm{oim} /+$, and $n=2 \mathrm{oim} / \mathrm{oim}$ ). These animals were allowed to survive to $25 \mathrm{wk}$ of age to assess recovery from the radiographically evident osteopetrotic features and were then killed. Due to the observational nature of the study, some of the groups had inadequate numbers of genotype and treatment matched controls. Therefore, statistical analysis could not be performed, and thus, the majority of the results are presented in case report format. Quantitative results were reported for $+/+$ and oim/oim mice only, but the findings for the oim/+ mice were similar.

\section{Analysis of Weight Gain}

Animals were weighed twice weekly during treatment, and weekly during the recovery portion of the experiment.

\section{Serum Chemistry}

TRACP-5b Assay. Blood was collected monthly in serum separator tubes (retro-orbital puncture under isoflurane anesthesia) and at the time of killing (cardiac puncture). Samples were centrifuged for $30 \mathrm{~min}$ and frozen at $-80^{\circ} \mathrm{C}$ until analysis for osteoclast activity/ number via TRACP-5b assay at Amgen. The TRACP-5b assay is a solid-phase immunofixed enzyme activity assay for the determination of osteoclast-derived TRACP-5b activity in mouse serum (IDS, Fountain Hills, AZ).

\section{Radiographic Analyses}

Anteroposterior and lateral digital radiographs were obtained monthly under isofluorine sedation using an MX-20 Live Animal Faxitron (Faxitron X-Ray, Lincolnshire, IL) at $24 \mathrm{~V}$ for 10 s. Each Faxitron image included an aluminum alloy step-density standard for calibration.

\section{Histology}

At the time of killing, one femur per animal was fixed in formalin for $24-48 \mathrm{~h}$ and decalcified in 10\% EDTA ( $\mathrm{pH} 7.2-7.4$ ) for $2 \mathrm{wk}$. Tissues were embedded in paraffin, sectioned at $7 \mu \mathrm{m}$ thickness along the coronal plate from anterior to posterior, and stained with hematoxylin and eosin, Alcian blue (images are not included in the article due to space limitations), and tartrate-resistant acid phosphatase (41). Sections containing the distal end of the femur were evaluated by the pathologist (E.D.) without knowledge as to the treatment/recovery status of the animal.

\section{Dental Evaluation}

Hemi-mandibles of eight 15-wk-old animals (treated and control male or female animals of $+/+$, oim $/+$, and oim/oim background) were excised and analyzed by microcomputed tomography (microCT) for mineralization abnormalities. Micro-CT data were acquired on the hemi-jaws with a Scanco $\mu$ CT35 scanner (Scanco Medical, Brüttisellen, Switzerland), with a $12 \mu \mathrm{m}$ voxel size, a $0.36^{\circ}$ rotation step ( $180^{\circ}$ angular range), and a $400 \mathrm{~ms}$ exposure, two frames per view. The molar areas from the same hemi-mandible were scanned separately with a $6 \mu \mathrm{m}$ voxel size. The Scanco $\mu \mathrm{CT}$ software (HP, Palo Alto, CA, DECwindows Motif 1.6) was used for three-dimensional reconstruction and viewing of images.

\section{ACKNOWLEDGMENTS}

We thank Lyudmila Lukashova for assistance with micro-CT and Stephen Doty and the Analytical Microscopy Core for assistance with histology. 


\section{STATEMENT OF FINANCIAL SUPPORT}

Funding for this study was provided by a research grant from Amgen (20082009), and National Institutes of Health (NIH) grant AR48337. The micro-CT and analytical microscopy cores are components of the $\mathrm{NIH}$-supported Musculoskeletal Repair and Regeneration Core Center (AR046121). R.B. received funding from the Osteogenesis Imperfecta Foundation through a Michael Geisman fellowship.

\section{REFERENCES}

1. Cheung MS, Glorieux FH Osteogenesis Imperfecta: update on presentation and management. Rev Endocr Metab Dis 2008;9:153-60.

2. Rauch F, Munns C, Land C, Glorieux FH. Pamidronate in children and adolescents with osteogenesis imperfecta: effect of treatment discontinuation. J Clin Endocrinol Metab 2006;91:1268-74.

3. Munns CF, Rauch F, Zeitlin L, Fassier F, Glorieux FH. Delayed osteotomy but not fracture healing in pediatric osteogenesis imperfecta patients receiving pamidronate. J Bone Miner Res 2004;19:1779-86.

4. Land C, Rauch F, Travers R, Glorieux FH. Osteogenesis imperfecta type VI in childhood and adolescence: effects of cyclical intravenous pamidronate treatment. Bone 2007;40:638-44.

5. Whyte MP, Wenkert D, Clements KL, McAlister WH, Mumm S. Bisphosphonate-induced osteopetrosis. N Engl J Med 2003;349:457-63.

6. Kearns AE, Khosla S, Kostenuik PJ. Receptor activator of nuclear factor kappaB ligand and osteoprotegerin regulation of bone remodeling in health and disease. Endocr Rev 2008;29:155-92.

7. Brown JP, Prince RL, Deal C, et al. Comparison of the effect of denosumab and alendronate on BMD and biochemical markers of bone turnover in postmenopausal women with low bone mass: a randomized, blinded, phase 3 trial. J Bone Miner Res 2009;24:153-61.

8. Ellis GK, Bone HG, Chlebowski R, et al. Randomized trial of denosumab in patients receiving adjuvant aromatase inhibitors for nonmetastatic breast cancer. J Clin Oncol 2008;26:4875-82.

9. Dougall WC, Glaccum M, Charrier K, et al. RANK is essential for osteoclast and lymph node development. Genes Dev 1999;13:2412-24.

10. Kostenuik PJ, Nguyen HQ, McCabe J, et al. Denosumab, a fully human monoclonal antibody to RANKL, inhibits bone resorption and increases BMD in knock-in mice that express chimeric (murine/human) RANKL. J Bone Miner Res 2009;24:182-95.

11. Canon JR, Roudier M, Bryant R, et al. Inhibition of RANKL blocks skeletal tumor progression and improves survival in a mouse model of breast cancer bone metastasis. Clin Exp Metastasis 2008;25:119-29.

12. Delos D, Yang X, Ricciardi BF, Myers ER, Bostrom MP, Camacho NP. The effects of RANKL inhibition on fracture healing and bone strength in a mouse model of osteogenesis imperfecta. J Orthop Res 2008;26:153-64.

13. Kim HK, Morgan-Bagley S, Kostenuik P. RANKL inhibition: a novel strategy to decrease femoral head deformity after ischemic osteonecrosis. J Bone Miner Res 2006;21:1946-54.

14. Miller RE, Branstetter D, Armstrong A, et al. Receptor activator of NF-kappa B ligand inhibition suppresses bone resorption and hypercalcemia but does not affect host immune responses to influenza infection. J Immunol 2007;179:266-74.

15. Ominsky MS, Kostenuik PJ, Cranmer P, Smith SY, Atkinson JE. The RANKL inhibitor OPG-Fc increases cortical and trabecular bone mass in young gonad-intact cynomolgus monkeys. Osteoporos Int 2007;18:1073-82.

16. Ominsky MS, Li X, Asuncion FJ, et al. RANKL inhibition with osteoprotegerin increases bone strength by improving cortical and trabecular bone architecture in ovariectomized rats. J Bone Miner Res 2008;23:672-82.

17. Padagas J, Colloton M, Shalhoub V, et al. The receptor activator of nuclear factor-kappaB ligand inhibitor osteoprotegerin is a bone-protective agent in a rat model of chronic renal insufficiency and hyperparathyroidism. Calcif Tissue Int 2006;78:35-44.

18. Stolina M, Adamu S, Ominsky M, et al. RANKL is a marker and mediator of local and systemic bone loss in two rat models of inflammatory arthritis. J Bone Miner Res 2005;20:1756-65.

19. Zwerina J, Hayer S, Tohidast-Akrad M, et al. Single and combined inhibition of tumor necrosis factor, interleukin-1, and RANKL pathways in tumor necrosis factor-induced arthritis: effects on synovial inflammation, bone erosion, and cartilage destruction. Arthritis Rheum 2004;50:277-90.
20. Hsu H, Lacey DL, Dunstan CR, et al. Tumor necrosis factor receptor family member RANK mediates osteoclast differentiation and activation induced by osteoprotegerin ligand. Proc Natl Acad Sci USA 1999; 96:3540-5.

21. Askmyr MK, Fasth A, Richter J. Towards a better understanding and new therapeutics of osteopetrosis. Br J Haematol 2008;140:597-609.

22. Marks SC Jr, Lane PW. Osteopetrosis, a new recessive skeletal mutation on chromosome 12 of the mouse. J Hered 1976;67:11-8.

23. Yoshino M, Yamazaki H, Yoshida H, et al. Reduction of osteoclasts in a critical embryonic period is essential for inhibition of mouse tooth eruption. J Bone Miner Res 2003;18:108-16.

24. Bargman R, Posham R, Boskey AL, DiCarlo E, Raggio C, Pleshko N. Comparable outcomes in fracture reduction and bone properties with RANKL inhibition and alendronate treatment in a mouse model of osteogenesis imperfecta. Osteoporos Int 2012;23:1141-50.

25. Chipman SD, Sweet HO, McBride DJ Jr, et al. Defective pro alpha 2(I) collagen synthesis in a recessive mutation in mice: a model of human osteogenesis imperfecta. Proc Natl Acad Sci USA 1993;90:1701-5.

26. Camacho NP, Raggio CL, Doty SB, et al. A controlled study of the effects of alendronate in a growing mouse model of osteogenesis imperfecta. Calcif Tissue Int 2001;69:94-101.

27. Min H, Morony S, Sarosi I, et al. Osteoprotegerin reverses osteoporosis by inhibiting endosteal osteoclasts and prevents vascular calcification by blocking a process resembling osteoclastogenesis. J Exp Med 2000; 192:463-74

28. Kaczmarczyk-Sedlak I, Janiec W. Effects of catecholamines on blood pressure in the long bone marrow cavity in rats with bisphosphonate-induced osteopetrosis. Pharmacol Rep 2005;57:623-34.

29. Reynolds JJ, Murphy H, Mühlbauer RC, Morgan DB, Fleisch H. Inhibition by diphosphonates of bone resorption in mice and comparison with greylethal osteopetrosis. Calcif Tissue Res 1973;12:59-71.

30. Jin Q, Cirelli JA, Park CH, et al. RANKL inhibition through osteoprotegerin blocks bone loss in experimental periodontitis. J Periodontol 2007;78:1300-8.

31. Iotsova V, Caamaño J, Loy J, Yang Y, Lewin A, Bravo R. Osteopetrosis in mice lacking NF-kappaB1 and NF-kappaB2. Nat Med 1997;3:1285-9.

32. Kaczmarczyk-Sedlak I. The effects of pamidronate on mechanical properties, growth and structural changes in rat bones. Acta Pol Pharm 1995; 52:509-13.

33. Whyte MP, McAlister WH, Novack DV, Clements KL, Schoenecker PL, Wenkert D. Bisphosphonate-induced osteopetrosis: novel bone modeling defects, metaphyseal osteopenia, and osteosclerosis fractures after drug exposure ceases. J Bone Miner Res 2008;23:1698-707.

34. Lekic P, Rubbino I, Krasnoshtein F, Cheifetz S, McCulloch CA, Tenenbaum H. Bisphosphonate modulates proliferation and differentiation of rat periodontal ligament cells during wound healing. Anat Rec 1997;247:329-40.

35. Kawata T, Tokimasa C, Nowroozi N, et al. Lack of the bone remodeling in osteopetrotic (op/op) mice associated with microdontia. J Craniofac Genet Dev Biol 1999;19:113-7.

36. Niida S, Abe M, Suemune S, Yoshiko Y, Maeda N, Yamasaki A. Restoration of disturbed tooth eruption in osteopetrotic (op/op) mice by injection of macrophage colony-stimulating factor. Exp Anim 1997;46:95-101.

37. Heinrich J, Bsoul S, Barnes J, Woodruff K, Abboud S. CSF-1, RANKL and OPG regulate osteoclastogenesis during murine tooth eruption. Arch Oral Biol 2005;50:897-908.

38. Kamoun-Goldrat A, Ginisty D, Le Merrer M. Effects of bisphosphonates on tooth eruption in children with osteogenesis imperfecta. Eur J Oral Sci 2008;116:195-8.

39. Raghunath M, Bruckner P, Steinmann B. Delayed triple helix formation of mutant collagen from patients with osteogenesis imperfecta. J Mol Biol 1994;236:940-9.

40. Akatsu T, Murakami T, Ono K, et al. Osteoclastogenesis inhibitory factor exhibits hypocalcemic effects in normal mice and in hypercalcemic nude mice carrying tumors associated with humoral hypercalcemia of malignancy. Bone 1998;23:495-8.

41. Filgueira L. Fluorescence-based staining for tartrate-resistant acidic phosphatase (TRAP) in osteoclasts combined with other fluorescent dyes and protocols. J Histochem Cytochem 2004;52:411-4. 